\title{
Legionella pneumonia due to non- Legionella pneumophila serogroup 1: usefulness of the six-point scoring system
}

\author{
Akihiro Ito ${ }^{1^{*}}$, Tadashi Ishida', Yasuyoshi Washio ${ }^{1,2}$, Akio Yamazaki ${ }^{1}$ and Hiromasa Tachibana ${ }^{1,3}$
}

\begin{abstract}
Background: Because of a limited number of reports, we aimed to investigate the clinical characteristics of patients with Legionella pneumonia due to non-Legionella pneumophila serogroup 1 and the diagnostic usefulness of the six-point scoring system for such patients compared with patients with pneumonia caused by L. pneumophila serogroup 1.

Methods: We retrospectively analysed patients diagnosed with Legionella pneumonia due to non-L. pneumophila serogroup 1 between March 2001 and June 2016. We examined the clinical characteristics, including symptoms, laboratory findings, radiologic findings, pneumonia severity, initial treatment and prognosis. We also calculated scores using the six-point scoring system in these patients. Furthermore, we compared the clinical characteristics and six-point scores between non-L. pneumophila serogroup 1 patients and L. pneumophila serogroup 1 patients among hospitalized community-acquired pneumonia patients enrolled prospectively between October 2010 and July 2016.
\end{abstract}

Results: Eleven patients had pneumonia due to non-L. pneumophila serogroup 1; their median age was 66 years and 8 patients (72.7\%) were male. The most common pathogen was L. pneumophila serogroup 3 (6/11), followed by L. pneumophila serogroup 9 (3/11), L. pneumophila serogroup $6(1 / 11)$ and L. longbeachae (1/11). Non-specific symptoms, such as fever and cough, were common. Six patients (54.5\%) had liver enzyme elevation, but no patient developed hyponatraemia at $<130 \mathrm{mEq} / \mathrm{L}$. Nine patients (81.8\%) showed lobar pneumonia and 7 patients (63.6\%) manifested with consolidation and ground-glass opacity. Patients with mild to moderate severity comprised 10 (90.9\%) by CURB-65 and 5 (45.5\%) by the Pneumonia Severity Index. Of all patients, 4 were admitted to the intensive care unit and 3 died despite appropriate empiric therapy. The clinical characteristics were not significantly different between non-L. pneumophila serogroup 1 patients and L. pneumophila serogroup 1 patients $(n=23)$. At a cut-off value of $\geq 2$ points, the sensitivity of the six-point scoring system was $54.5 \%$ (6/11) for non-L. pneumophila serogroup 1 patients and 95.7\% (22/23) for L. pneumophila serogroup 1 patients.

Conclusions: Cases of non-L. pneumophila serogroup 1 pneumonia varied in severity from mild to severe and the clinical characteristics were often non-specific. The six-point scoring system was not useful in predicting such Legionella pneumonia cases.

Keywords: Legionella pneumonia, Legionella pneumophila serogroup 1, Community-acquired pneumonia, Diagnostic scoring system

\footnotetext{
* Correspondence: ai12306@kchnet.or.jp

${ }^{1}$ Department of Respiratory Medicine, Ohara Healthcare Foundation,

Kurashiki Central Hospital, Miwa 1-1-1, Kurashiki, Okayama 710-8602, Japan

Full list of author information is available at the end of the article
} 


\section{Background}

Legionella pneumonia is a type of pulmonary infection that is caused by Gram-negative bacilli and is an important cause of severe community-acquired pneumonia (CAP) [1]. The most frequently identified causative pathogen of Legionella pneumonia is Legionella pneumophila serogroup $1[2,3]$, probably because it can be quickly diagnosed by the urinary antigen test. However, about $20 \%$ of Legionella pneumonia cases due to non-L. pneumophila serogroup 1 are diagnosed not by the urinary antigen test, but by culture using Wadowsky-Yee-Okuda (WYO)- $\alpha$ or Buffered Charcoal Yeast Extract (BCYE)- $\alpha$ medium [2, 3]. Legionella pneumonia due to non-L. pneumophila serogroup 1 can be both severe [4-8] and mild to moderate $[9,10]$.

To date, there have been few reports that summarised the clinical characteristics of such cases, including symptoms, laboratory findings, radiologic findings, pneumonia severity, treatment and prognosis. To predict the probability of Legionella pneumonia, Fiumefreddo et al. [11] proposed a six-point scoring system using dichotomised routine clinical and laboratory variables. Haubitz et al. [12] validated the usefulness of this scoring system using a large multinational database. However, to our knowledge, there are no reports evaluating the usefulness of this scoring system in predicting non-L. pneumophila serogroup 1 Legionella pneumonia. This study aimed to investigate the clinical characteristics of Legionella pneumonia due to non-L. pneumophila serogroup 1 and the usefulness of the six-point scoring system in predicting such cases compared with those of L. pneumophila serogroup 1.

\section{Methods}

\section{Study design and setting}

This study retrospectively analysed hospitalised and outpatient CAP cases due to Legionella species other than L. pneumophila serogroup 1 at Kurashiki Central Hospital between March 2001 and June 2016. CAP was diagnosed in accordance with the latest Infectious Diseases Society of America/American Thoracic Society guidelines [13] as follows: at least one clinical symptom (fever, cough, sputum production, dyspnoea or pleuritic chest pain) plus at least more than one finding of elevated inflammatory biomarkers or coarse crackles on auscultation, in addition to new infiltrate on chest radiography. Exclusion criteria were age $<15$ years, acquired immune deficiency syndrome, hospital-acquired pneumonia and healthcare-associated pneumonia [14]. A total of 11 patients with Legionella pneumonia due to non-L. pneumophila serogroup 1 were finally included in this analysis. This study was approved by the institutional review board of Kurashiki Central Hospital (approval number 2364). Based on the Ethical Guidelines for Medical and Health Research Involving Human Subjects of the Ministry of Health, Labour and Welfare, we notify the research subjects of, or make public, information concerning the research on the web. All patients gave their informed consent to participate in this study by being given opportunities to refuse to participate.

In all patients, the severity of pneumonia was assessed on admission or on the first visit using the Pneumonia Severity Index (PSI) [15] and CURB-65 score [confusion, urea $>7 \mathrm{mmol} / \mathrm{L}$, respiratory rate $\geq 30$ breaths $/ \mathrm{min}$, low blood pressure (systolic $<90 \mathrm{mmHg}$ or diastolic $\leq 60 \mathrm{mmHg}$ ) and age $\geq 65$ years)] [16]. All patients were administered antimicrobial agents based on the decision of the physician in charge and in accordance with the recommendations of the CAP guidelines of the Japanese Respiratory Society [17]. Blood tests and chest X-ray images were examined to assess the effectiveness of the antimicrobials as appropriate. We treated patients in the intensive care unit (ICU) if they needed mechanical ventilatory support and/or vasopressor drugs.

In all patients, the variables examined included age, sex, comorbidity, smoking history, symptoms, bacterial strain, chest X-ray or computed tomography (CT) findings, laboratory findings, CURB-65 and PSI severity scores, initial treatment and prognosis.

\section{Microbiologic investigation}

On admission, sputum and blood for cultures, serum for measuring antibodies and urine for Streptococcus pneumoniae or L. pneumophila antigen test were collected to detect as clearly as possible the causative microorganisms of CAP. Legionella species was identified using culture on WYO- $\alpha$ medium, not by polymerase chain reaction. We diagnosed Legionella pneumonia due to non-L. pneumophila serogroup 1 based on sputum or blood culture.

\section{Radiologic findings}

Radiologic tests were examined by chest X-ray and/or $\mathrm{CT}$, which were interpreted by four pulmonologists. We examined the affected lobes, radiologic pattern and the presence of consolidation, ground-glass opacity (GGO), nodule, pleural effusion, cavity or lymphadenopathy. We defined airspace consolidation as infiltration with obscured vascular margins and GGO as an increase in hazy attenuation with intact vascular markings. Mediastinal lymphadenopathy was defined as the presence of lymph nodes greater than $10 \mathrm{~mm}$ in minimal diameter.

\section{Clinical characteristics of Legionella pneumonia due to non-L. pneumophila serogroup 1 and $L$. pneumophila serogroup 1}

We also investigated clinical characteristics including age, sex, smoking history, vital signs, laboratory findings 
and pneumonia severity in Legionella pneumonia due to L. pneumophila serogroup 1 patients and compared them with those of non-L. pneumophila serogroup 1 patients. L. pneumophila serogroup 1 patients were diagnosed by the urinary antigen test and/or sputum culture or serum antibody. These patients were part of a prospective, observational cohort at our hospital between October 2010 and July 2016 (UMIN000004353). All patients with Legionella pneumonia due to $L$. pneumophila serogroup 1 also gave their informed consent to participate in this study.

\section{The six-point scoring system for the diagnosis of Legionella pneumonia}

The six-point scoring system was determined in each case and comprised dichotomised routine clinical and laboratory variables, including fever $>39.4{ }^{\circ} \mathrm{C}$, C-reactive protein (CRP) value $>187 \mathrm{mg} / \mathrm{L}$, lactate dehydrogenase $>225 \mathrm{mmol} / \mathrm{L}$, thrombocytopenia $<171 \times 10^{9} / \mathrm{L}$, hyponatraemia (serum sodium $<133 \mathrm{mmol} / \mathrm{L}$ ) and unproductive cough. The six-point scores of non-L. pneumophila serogroup 1 patients were compared with those of $L$. pneumophila serogroup 1 patients.

\section{Statistical analysis}

Categorical variables are expressed as frequency (percentage) and continuous variables are expressed as medians and interquartile ranges (IQR). Categorical variables were analysed using Fisher's exact test and continuous variables were analysed by the nonparametric Mann-Whitney U-test. A $P$ value of < 0.05 was considered significant. Statistical analyses were performed using $\mathrm{R}$ (version 3.0.3, Vienna, Austria).

\section{Results}

Patients' characteristics

The characteristics of the study population are shown in Table 1. Cases 1, 2, 6 and 9 were outpatients and the others were inpatients. The age range was 58-82 years. Eight patients (72.7\%) were male. Diabetes mellitus, chronic liver disease and malignant disease were seen in 2 patients (18.2\%) each and 6 patients (54.5\%) had a smoking history ( 3 were past, 3 were current). The most common symptom was fever $(72.7 \%)$, followed by cough (54.5\%) and sputum production (54.5\%). Disturbance in consciousness was seen in only 1 patient; no patient had digestive symptoms, such as abdominal pain, vomiting and diarrhoea. The most common bacterial strain was $L$. pneumophila serogroup 3 (54.5\%), followed by $L$. pneumophila serogroup 9 (27.3\%), L. pneumophila serogroup 6 (9.1\%) and L. longbeachae (9.1\%).

\section{Radiologic findings}

Table 2 shows the chest X-ray and CT findings. Chest CT scan was available for all patients except one. There were 4 patients (Cases 3, 5, 8 and 9) with unilobar infiltration and 7 patients (Cases 1, 2, 4, 6, 7, 10 and 11) with multilobar infiltration. All patients, except two (Cases 1 and 2) (81.8\%), had lobar pneumonia. The most common finding was GGO (81.8\%), followed by consolidation $(72.7 \%)$. Consolidation plus GGO was seen in 7 patients (63.6\%). Nodule and pleural effusion were found in 3 patients $(27.3 \%)$ each; there was no cavitary lesion in any of the patients.

\section{Laboratory findings}

Table 3 shows the laboratory findings in all non- $L$. pneumophila serogroup 1 patients. High CRP concentration was found in 9 patients $(81.8 \%)$; a white blood cell count $>10 \times 10^{3} / \mu \mathrm{L}$ was seen in only 3 patients

Table 1 Characteristics of all patients with Legionella pneumonia due to non-Legionella pneumophila serogroup 1

\begin{tabular}{lllllll}
\hline & Age $(\mathrm{y})$ & Sex & Comorbidity & Smoking history & Symptoms & Legionella species \\
\hline 1 & 58 & F & None & Never & Cough, sputum & L. pneumophila SG3 \\
2 & 58 & F & RA, steroid use & Unknown & Cough, sputum & L. pneumophila SG3 \\
3 & 59 & M & DM, CLD, heavy drinker & Current & Fever, consciousness disturbance & L. pneumophila SG9 \\
4 & 60 & F & Malignant disease & Unknown & Fever & L. pneumophila SG3 \\
5 & 65 & M & None & Past & Fever, myalgia, arthralgia & L. pneumophila SG9 \\
6 & 66 & M & DM, CLD & Past & Fever, cough, sputum & L. pneumophila SG3 \\
7 & 68 & M & None & Current & Fever, dyspnoea & L. longbeachae \\
8 & 71 & M & None & Unknown & Fever, cough, sputum, arthralgia & L. pneumophila SG6 \\
9 & 74 & M & Malignant disease & Past & Fever, cough, sputum, haemoptysis, dyspnoea & L. pneumophila SG3 \\
10 & 77 & $M$ & COPD, asthma & Past & Dyspnoea & L. pneumophila SG3 \\
11 & 82 & $M$ & None & Unknown & Fever, cough, sputum, dyspnoea & L. pneumophila SG9 \\
\hline
\end{tabular}

Abbreviations: CLD chronic liver disease, COPD chronic obstructive pulmonary disease, DM diabetes mellitus, RA rheumatoid arthritis, SG serogroup 
Table 2 Chest imaging findings in all patients with Legionella pneumonia due to non-Legionella pneumophila serogroup 1

\begin{tabular}{|c|c|c|c|c|c|c|c|c|c|}
\hline & Modality & Affected Lobes & Radiologic pattern & Consolidation & GGO & Nodule & Effusion & Cavity & Lymph node swelling \\
\hline 1 & CT & LUL, LLL & Lobular & - & - & + & - & - & + \\
\hline 2 & CT & RUL, RML, LUL & Lobular & - & + & + & - & - & - \\
\hline 3 & $\mathrm{CT}$ & LUL & Lobar & + & + & - & + & - & - \\
\hline 4 & CT & RUL, RML, RLL, LUL, LLL & Lobar & + & + & - & - & - & - \\
\hline 5 & $x p$ & LLL & Lobar & + & + & - & - & - & - \\
\hline 6 & CT & RUL, RLL, LLL & Lobar & - & + & + & - & - & - \\
\hline 7 & $\mathrm{CT}$ & RUL, RLL, LUL, LLL & Lobar & + & + & - & + & - & + \\
\hline 8 & CT & LUL & Lobar & + & - & - & - & - & - \\
\hline 9 & CT & RUL & Lobar & + & + & - & + & - & + \\
\hline 10 & $\mathrm{CT}$ & LUL, LLL & Lobar & + & + & - & - & - & - \\
\hline 11 & CT & RUL, RML, RLL, LUL, LLL & Lobar & + & + & - & - & - & - \\
\hline
\end{tabular}

Abbreviations: CT computed tomography, GGO ground-glass opacity, LLL left lower lobe, LUL left upper lobe, RLL right lower lobe, RML right middle lobe, RUL right upper lobe, $X p$ radiograph

(27.3\%). Elevated liver enzyme, either aspartate aminotransferase or alanine aminotransferase, was seen in 6 patients (54.5\%). Hyponatraemia was seen in only 3 patients (27.3\%). Among 4 patients in whom creatine phosphokinase (CPK) was measured, $2(50 \%)$ had high values.

\section{Severity of pneumonia, initial treatment and prognosis}

Table 4 shows the pneumonia severity, initial treatment and prognosis in all non-L. pneumophila serogroup 1 patients. Most patients $(10 / 11,90.9 \%)$ had mild to moderate pneumonia by CURB-65 ( $\leq 2$ points) and about half $(5 / 11,45.5 \%)$ had mild to moderate pneumonia by PSI (sclass III). Four patients (36.4\%) were treated in the ICU. All patients were administered empiric antimicrobials that covered Legionella species; however, 3 patients (27.3\%) died.
Clinical characteristics of non-L. pneumophila serogroup 1 and $L$. pneumophila serogroup 1 patients

Between October 2010 and July 2016, we prospectively enrolled 1236 hospitalized CAP patients, of which 23 patients had Legionella pneumonia due to L. pneumophila serogroup 1. Eleven patients were diagnosed by using the urinary antigen test and sputum culture, 11 patients with only the urinary antigen test and 1 patient with serum antibody. Clinical characteristics including age, sex, comorbidities, vital signs, laboratory examinations and severity scores between non-L. pneumophila serogroup 1 patients and $L$. pneumophila serogroup 1 patients were not significantly different (Table 5). The frequency of ICU admission was not significantly different between these two groups, however, in-hospital mortality was significantly higher in non- $L$. pneumophila serogroup 1 patients than L. pneumophila serogroup 1 patients $(27.3 \%$ vs $0 \%, P=0.03)$.

Table 3 Laboratory findings in all patients with Legionella pneumonia due to non-Legionella pneumophila serogroup 1

\begin{tabular}{|c|c|c|c|c|c|c|c|c|c|c|c|c|}
\hline & $\begin{array}{l}\text { WBC } \\
\left(\times 10^{3} / \mu \mathrm{L}\right)\end{array}$ & $\begin{array}{l}\text { CRP } \\
(\mathrm{mg} / \mathrm{L})\end{array}$ & $\begin{array}{l}\text { TP } \\
(\mathrm{g} / \mathrm{dL})\end{array}$ & $\begin{array}{l}\text { Alb } \\
\text { (g/dL) }\end{array}$ & $\begin{array}{l}\text { AST } \\
(\mathrm{IU} / \mathrm{L})\end{array}$ & $\begin{array}{l}\text { ALT } \\
(\mathrm{IU} / \mathrm{L})\end{array}$ & $\begin{array}{l}\mathrm{LDH} \\
(\mathrm{IU} / \mathrm{L})\end{array}$ & $\begin{array}{l}\text { BUN } \\
\text { (mg/dL) }\end{array}$ & $\begin{array}{l}\mathrm{Cr} \\
(\mathrm{mg} / \mathrm{dL})\end{array}$ & $\begin{array}{l}\mathrm{Na} \\
(\mathrm{mEq} / \mathrm{L})\end{array}$ & $\begin{array}{l}\text { Plt } \\
\left(\times 10^{9} / \mathrm{L}\right)\end{array}$ & $\begin{array}{l}\text { CPK } \\
\text { (IU/L) }\end{array}$ \\
\hline 1 & 6.6 & 1.1 & 8.3 & 4.4 & 21 & 13 & 195 & 15 & 0.67 & 139 & 304 & ND \\
\hline 2 & 8.8 & 0.6 & 6.2 & 3.6 & 32 & 35 & 214 & 23 & 0.80 & 141 & 226 & ND \\
\hline 3 & 7.3 & 328.6 & 6.2 & 2.5 & 636 & 274 & 1309 & 23 & 1.5 & 131 & 197 & 9757 \\
\hline 4 & 5.9 & 174.7 & 3.3 & 1.3 & 33 & 20 & 421 & 51 & 2.74 & 142 & 13 & ND \\
\hline 5 & 8.2 & 195.1 & 5.9 & 2.7 & 49 & 36 & 256 & 13 & 0.80 & 130 & 202 & 167 \\
\hline 6 & 6.9 & 222 & 7.3 & 2.9 & 30 & 45 & 204 & 15 & 0.95 & 136 & 261 & ND \\
\hline 7 & 14.8 & 345.4 & 5.1 & 2.2 & 50 & 29 & 381 & 14 & 0.69 & 133 & 215 & 61 \\
\hline 8 & 10.2 & 125 & 6.7 & 2.7 & 17 & 4 & 314 & 12 & 0.7 & 137 & 323 & ND \\
\hline 9 & 8.3 & 266.3 & 5.8 & 2.5 & 22 & 20 & 117 & 12 & 0.82 & 131 & 120 & ND \\
\hline 10 & 9.6 & 5.6 & 6.9 & 3.6 & 24 & 21 & 198 & 13 & 0.85 & 141 & 190 & ND \\
\hline 11 & 14.1 & 282 & 7.1 & 3.5 & 190 & 60 & 885 & 34 & 1.6 & 137 & 132 & 6728 \\
\hline
\end{tabular}


Table 4 Severity of pneumonia, treatment and prognosis in all non-Legionella pneumophila serogroup 1 patients

\begin{tabular}{|c|c|c|c|c|c|}
\hline & CURB-65 (points) & PSI (class) & Initial treatment & ICU admission & Outcome \\
\hline 1 & 0 & $\|$ & AZM-PO & - & Survived \\
\hline 2 & 0 & $\|$ & AZM-PO & - & Survived \\
\hline 3 & 2 & IV & LVFX-IV, AZM-IV & + & Survived \\
\hline 4 & 1 & IV & MEPM, CPFX-IV & + & Died \\
\hline 5 & 2 & IV & CTRX, AZM-PO & - & Survived \\
\hline 6 & 1 & III & AZM-PO & - & Survived \\
\hline 7 & 2 & IV & CTRX, LVFX-IV & + & Died \\
\hline 8 & 1 & III & LVFX-PO & - & Survived \\
\hline 9 & 1 & IV & LVFX-PO & - & Survived \\
\hline 10 & 2 & III & LVFX-PO & - & Survived \\
\hline 11 & 4 & V & MEPM, CPFX-IV & + & Died \\
\hline
\end{tabular}

Abbreviations: $A Z M$ azithromycin, CPFX ciprofloxacin, CTRX ceftriaxone, CURB-65 confusion, urea $>7 \mathrm{mmol} / \mathrm{L}$, respiratory rate $\geq 30$ breaths/min, low blood pressure (systolic $<90 \mathrm{mmHg}$ or diastolic $\leq 60 \mathrm{mmHg}$ ) and age $\geq 65$ years, ICU intensive care unit, IV intravenous, LVFX levofloxacin, MEPM meropenem, PO per os, PSI Pneumonia Severity Index

\section{The six-point scoring system for predicting Legionella pneumonia}

The six-point scores for predicting Legionella pneumonia due to non-L. pneumophila serogroup 1 are shown in Table 6 and those for predicting Legionella pneumonia due to $L$. pneumophila serogroup 1 are shown in Additional file 1: Table S1. The median six-point score was significantly lower in non-L. pneumophila serogroup 1 patients than in $L$. pneumophila serogroup 1 patients (2.0, IQR $0.5-3.0$ vs. 3.0 , IQR $2.0-3.5 ; P=0.021$ ). At a cut-off value of $\geq 2$ points, the sensitivity of this scoring system was $54.5 \%(6 / 11)$ for non-L. pneumophila serogroup 1 patients and $95.7 \%$ (22/23) for L. pneumophila serogroup 1 patients.

\section{Discussion}

This study on 11 patients demonstrated the clinical characteristics of Legionella pneumonia due to non- $L$. pneumophila serogroup 1. Legionella pneumonia is a principal cause of severe CAP [18], particularly among patients $<60$ years, in whom the rate of severe pneumonia due to Legionella species was reported to be as high as $14.1 \%$ compared with $2.3 \%$ in patients $\geq 60$ years [1]. In this study, almost all patients had Legionella pneumonia of mild to moderate severity by CURB-65, and 4 cases could be treated as outpatients; however, 4 patients were admitted to the ICU and 3 of those died despite appropriate empiric therapy.

The reported comorbidities that could predispose one to Legionella pneumonia were chronic lung disease [19], glucocorticoid treatment [20], haematologic malignancies under chemotherapy [21] and solid tumours [22]. In this study, 1 patient had chronic obstructive pulmonary disease, 1 received steroid treatment and 2 had malignant disease; however, almost half of the patients $(5 / 11$, 45.5\%) had no comorbidities.
Cunha et al. reported that Legionella pneumonia presented with non-specific symptoms of fever $>38.8{ }^{\circ} \mathrm{C}$ in $67-100 \%$, cough in $41-92 \%$, chills in $15-77 \%$ and dyspnoea in $36-56 \%$, as well as relatively specific symptoms of neurologic abnormalities in 38-53\%, myalgia or arthralgia in $20-43 \%$, diarrhoea in $19-47 \%$, chest pain in $14-50 \%$, headache in $17-43 \%$ and nausea or vomiting in 9-25\% [23]. In our case series of Legionella pneumonia, many patients manifested with fever $(8 / 11,72.7 \%)$ and cough $(6 / 11,54.5 \%)$, but only a few had arthralgia or myalgia $(2 / 11,18.2 \%)$ and disturbance in consciousness $(1 / 11,9.1 \%)$. No patient had specific symptoms of diarrhoea, nausea or vomiting.

For laboratory findings, hepatic dysfunction, hyponatraemia [24] and CPK elevation [25] were indicated as more significant findings in Legionella pneumonia patients than in those with pneumonia from other aetiology. Almost half of our patients $(5 / 11,45.5 \%)$ had normal liver enzymes, no patient had serum sodium < $130 \mathrm{mEq} / \mathrm{L}$ and only 2 of 4 had CPK elevation. The number of patients who did not have liver enzyme elevation and hyponatraemia was 5 of 11 patients (45.5\%). From these patterns of clinical symptoms and laboratory findings, Legionella pneumonia seemed difficult to predict in these patients.

The usefulness of the six-point scoring system proposed by Fiumefreddo et al. [11] in predicting Legionella pneumonia was validated by Haubitz et al. [12]. They showed that a score of $\geq 5$ had very high specificity of 99.0\% (95\% CI, 98.4-99.4) and high positive predictive value of $17.4 \%$ (95\% CI, 5.0-38.8), whereas a score of $<2$ had high sensitivity of $94.4 \%$ (95\% CI, 81.3-99.3) and high negative predictive value of $99.6 \%$ (95\% CI, 98.6-100). In this study, at a cut-off value of $\geq 2$ points, the sensitivity of this scoring system was $54.5 \%(6 / 11)$ for non- $L$. pneumophila serogroup 1 patients and 95.7\% (22/23) for 
Table 5 Clinical characteristics of non-Legionella pneumophila serogroup 1 and Legionella pneumophila serogroup 1 patients

\begin{tabular}{|c|c|c|c|}
\hline & non-L. pneumophila serogroup $1(n=11)$ & L. pneumophila serogroup $1(n=23)$ & $P$ value \\
\hline Age (y) & $66.0(59.5-72.5)$ & $70.0(58.5-74.5)$ & 0.96 \\
\hline Male & $8(72.7)$ & $21(91.3)$ & 0.30 \\
\hline \multicolumn{4}{|l|}{ Smoking history } \\
\hline Current + Past & $5(62.5)$ & $17(73.9)$ & 0.66 \\
\hline \multicolumn{4}{|l|}{ Comorbidity } \\
\hline Chronic heart disease & $0(0)$ & $7(30.4)$ & 0.07 \\
\hline COPD & $1(9.1)$ & $0(0)$ & 0.32 \\
\hline Diabetes mellitus & $2(18.2)$ & $7(30.4)$ & 0.68 \\
\hline Cerebrovascular disease & $0(0)$ & $5(21.7)$ & 0.15 \\
\hline Malignant disease & $2(18.2)$ & $2(8.7)$ & 0.58 \\
\hline Chronic kidney disease & $0(0)$ & $3(13.0)$ & 0.54 \\
\hline Chronic liver disease & $2(18.2)$ & $0(0)$ & 0.1 \\
\hline \multicolumn{4}{|l|}{ Vital signs } \\
\hline Body temperature $\left({ }^{\circ} \mathrm{C}\right)$ & 37.7 (36.9-38.6) & $38.8(37.8-39.5)$ & 0.06 \\
\hline Systolic blood pressure $(\mathrm{mmHg})$ & $121(121-130)$ & $136(122-157)$ & 0.11 \\
\hline Heart rate (beats/min) & $95(86-117)$ & 100 (87-104) & 0.82 \\
\hline \multicolumn{4}{|l|}{ Laboratory examinations } \\
\hline Alb (g/dL) & $2.7(2.5-3.6)$ & $3.0(2.3-3.3)$ & 0.66 \\
\hline $\mathrm{LDH}(\mathrm{U} / \mathrm{L})$ & $256(201-401)$ & $395(276-540)$ & 0.12 \\
\hline BUN (mg/dL) & $15(13-23)$ & $24(16-41)$ & 0.14 \\
\hline $\mathrm{Cr}(\mathrm{mg} / \mathrm{dL})$ & $0.82(0.75-1.23)$ & $1.06(0.83-1.74)$ & 0.12 \\
\hline $\mathrm{Na}(\mathrm{mmol} / \mathrm{L})$ & $137(132-140)$ & $134(130-137)$ & 0.14 \\
\hline $\mathrm{Ht}(\%)$ & $39.7(33.2-42.1)$ & 38.9 (35.2-41.8) & 0.90 \\
\hline Plt $\left(\times 10^{4} / \mu \mathrm{L}\right)$ & $20.2(16.1-24.4)$ & $16.4(14.2-19.8)$ & 0.16 \\
\hline $\mathrm{WBC}\left(\times 10^{3} / \mu \mathrm{L}\right)$ & $8.3(7.1-9.9)$ & $10.4(8.5-11.9)$ & 0.11 \\
\hline CRP (mg/L) & $195(65-274)$ & $230(184-281)$ & 0.31 \\
\hline CURB-65 (score) & & & 0.48 \\
\hline 0 & $2(18.2)$ & $2(8.7)$ & \\
\hline 1 & $4(36.4)$ & $7(30.4)$ & \\
\hline 2 & $4(36.4)$ & $8(34.8)$ & \\
\hline 3 & $0(0)$ & $5(21.7)$ & \\
\hline 4 & $1(9.1)$ & $1(4.3)$ & \\
\hline 5 & $0(0)$ & $0(0)$ & \\
\hline PSI (score) & $99(79-116)$ & $97(84-121)$ & 0.73 \\
\hline PSI (class) & & & 0.84 \\
\hline । & $0(0)$ & $1(4.3)$ & \\
\hline । & $2(18.2)$ & $2(8.7)$ & \\
\hline III & $3(27.3)$ & $6(26.1)$ & \\
\hline IV & $5(45.5)$ & $9(39.1)$ & \\
\hline v & $1(9.1)$ & $5(21.7)$ & \\
\hline ICU admission & $4(36.4)$ & $6(26.1)$ & 0.69 \\
\hline In-hospital mortality & $3(27.3)$ & $0(0)$ & 0.03 \\
\hline
\end{tabular}

Data are presented as medians (interquartile range) or $\mathrm{n}(\%)$

Abbreviations: Alb albumin, BUN blood urea nitrogen, COPD chronic obstructive pulmonary disease, $C r$ creatinine, CRP C-reactive protein, CURB-65 confusion, urea $>7 \mathrm{mmol} / \mathrm{L}$, respiratory rate $\geq 30$ breaths/min, low blood pressure (systolic $<90 \mathrm{mmHg}$ or diastolic $\leq 60 \mathrm{mmHg}$ ) and age $\geq 65 \mathrm{years,}$ $H t$ haematocrit, ICU intensive care unit, LDH lactate dehydrogenase, Na sodium, Plt platelet, PSI Pneumonia Severity Index, WBC white blood cell 
Table 6 The six-point scoring system for predicting Legionella pneumonia in non-Legionella pneumophila serogroup 1 patients

\begin{tabular}{|c|c|c|c|c|c|c|c|}
\hline & CRP $>187 \mathrm{mg} / \mathrm{L}$ & $\mathrm{Na}<133 \mathrm{mmol} / \mathrm{L}$ & Temperature $^{\mathrm{a}}>39.4{ }^{\circ} \mathrm{C}\left({ }^{\circ} \mathrm{C}\right)$ & Plt $<171 \times 10^{9} / \mathrm{L}$ & $\mathrm{LDH}>225 \mathrm{IU} / \mathrm{L}$ & Dry cough & Total score \\
\hline 1 & - & - & - (37.0) & - & - & - & 0 \\
\hline 2 & - & - & $-(36.5)$ & - & - & - & 0 \\
\hline 3 & + & + & - (39.2) & - & + & - & 3 \\
\hline 4 & - & - & - (38.8) & + & + & - & 2 \\
\hline 5 & + & + & - $(37.7)$ & - & + & - & 3 \\
\hline 6 & + & - & - (36.7) & - & - & - & 1 \\
\hline 7 & + & - & $-(38.3)$ & - & + & - & 2 \\
\hline 8 & - & - & - (38.3) & - & + & - & 1 \\
\hline 9 & + & + & - (37.0) & + & - & - & 3 \\
\hline 10 & - & - & - (36.5) & - & - & - & 0 \\
\hline 11 & + & - & - (39.3) & + & + & - & 3 \\
\hline
\end{tabular}

Abbreviations: CRP C-reactive protein, $L D H$ lactate dehydrogenase, Na sodium, Plt platelet

${ }^{\text {aThe }}$ patient's body temperature is indicated in parentheses

L. pneumophila serogroup 1 patients. This finding indicated that we can rule out Legionella pneumonia due to $L$. pneumophila serogroup 1 by using this scoring system, whereas about half of patients with Legionella pneumonia due to non-L. pneumophila serogroup 1 could have been misdiagnosed by this scoring system. Namely, Legionella pneumonia due to non- $L$. pneumophila serogroup 1 cannot be ruled out by the six-point scoring system.

$\mathrm{Yu}$ et al. reported that consolidation and GGO were the main CT findings in 23 Legionella pneumonia patients and almost all patients $(82.6 \%)$ had non-segmental distribution [26]. Sakai et al. showed that among 38 patients, consolidation plus GGO was seen in 35 (92.1\%), pleural effusion was seen in $23(60.5 \%)$ and ipsilateral hilar and/or mediastinal lymphadenopathy was seen in 17 (44.7\%). They also indicated that sharply demarcated peribronchovascular foci of consolidation intermingled with GGO was a significant finding in Legionella pneumonia compared with S. pneumoniae pneumonia [27]. Similarly, our study showed that consolidation $(72.7 \%)$ and GGO (81.8\%) were common radiologic findings that were seen in $63.6 \%$ of patients. However, findings of pleural effusion $(27.3 \%)$ and lymphadenopathy $(27.3 \%)$ were relatively less common in our study than in the previous reports.

Legionella pneumonia due to non-L. pneumophila serogroup 1 may vary in severity from mild or moderate, which could be treated in an outpatient setting, to severe, which needs ICU admission and has poor prognosis. Only a few of these patients showed specific symptoms of Legionella pneumonia, such as neurologic abnormalities, myalgia or arthralgia, headache, diarrhoea and nausea or vomiting. In addition, abnormalities in laboratory tests, such as liver enzyme elevation and hyponatraemia, were not usual. Therefore, the six-point scoring system for Legionella pneumonia might not be useful for predicting cases due to non-L. pneumophila serogroup 1. Although this scoring system did not include radiologic findings, some previous reports and the present study showed that a combination of consolidation and GGO was a significant finding that might suggest the aetiology of pneumonia as Legionella. Therefore, in CAP patients showing consolidation plus GGO on chest CT and those with resistance to $\beta$ lactam antibiotic therapy, performing sputum culture for Legionella species on WYO- $\alpha$ or BCYE- $\alpha$ medium might be helpful. Furthermore, we should consider administration of antibiotics that are effective for Legionella species despite a negative Legionella urinary antigen test.

This study had some limitations. First, the study was retrospective and included a small number of patients. Second, in all cases due to non- $L$. pneumophila serogroup 1, Legionella species was identified by culture, which is not routine for all CAP patients; therefore, there might have been bias. Nevertheless, these results were important in providing data on the clinical characteristics of Legionella pneumonia due to non-L. pneumophila serogroup 1 and the usefulness of the six-point scoring system for predicting Legionella pneumonia due to non- $L$. pneumophila serogroup 1.

\section{Conclusions}

In conclusion, most cases of Legionella pneumonia due to non-L. pneumophila serogroup 1 in this series presented as mild to moderate in severity. The six-point scoring system was not useful in predicting such cases. Therefore, urinary antigen test to detect non-L. pneumophila serogroup 1 would be expected in the future.

\section{Additional file}

Additional file 1: Table S1. The six-point scoring system in patients with Legionella pneumonia due to L. pneumophila serogroup 1. (DOCX $19 \mathrm{~kb}$ ) 


\section{Abbreviations}

Alb: albumin; BCYE-a: buffered charcoal yeast extract-a; BUN: blood urea nitrogen; CAP: community-acquired pneumonia; COPD: chronic obstructive pulmonary disease; CPK: creatine phosphokinase; Cr: creatinine; CRP: Creactive protein; CT: computed tomography; CURB-65: confusion, urea > $7 \mathrm{mmol} / \mathrm{L}$, respiratory rate $\geq 30$ breaths/min, low blood pressure (systolic < $90 \mathrm{mmHg}$ or diastolic $\leq 60 \mathrm{mmHg}$ ) and age $\geq 65$ years; GGO: ground-glass opacity; Ht: haematocrit; ICU: intensive care unit; IQR: interquartile range; LDH: lactate dehydrogenase; Na: sodium; Plt: platelet; PSI: Pneumonia Severity Index; WBC: white blood cell; WYO-a: Wadowsky-Yee-Okuda-a

\section{Acknowledgements}

We would like to thank all of our colleagues who recruited and treated the patients. We thank Hiroyuki Fujii from the Department of Clinical Laboratory, Ohara Healthcare Foundation, Kurashiki Central Hospital, for performing sputum culture for Legionella species identification. We thank Dr. Hiroshi Nakajima from the Department of Bacteriology, Okayama Prefectural Institute for Environmental Science and Public Health, and Dr. Junko AmemuraMaekawa from the Department of Bacteriology I, National Institute of Infectious Diseases, for performing Legionella species identification.

This study was awarded the 11th Award in the Category of Clinical Research Conferred by the Director of the West Japan Branch of the Japanese Society of Chemotherapy at the 64th Japanese Society of Chemotherapy West Japan branch general meeting held in November 2016.

\section{Funding}

No financial support was received for this study.

\section{Availability of data and materials}

The datasets used and analysed in the current study are available from the corresponding author upon request.

\section{Authors' contributions}

Al served as the principal author, had full access to all data in the study and takes responsibility for the integrity and accuracy of the data and data analysis. $\mathrm{Al}$ and $\mathrm{Tl}$ contributed to the study conception and design. $\mathrm{Al}, \mathrm{Tl}$, YW, AY and HT contributed to acquisition of data. Al, TI and HT contributed to analysis and interpretation of data. Al, TI, YW, AY and HT contributed to drafting and revision of the manuscript and approval of the final version to be submitted for consideration for publication.

\section{Ethics approval and consent to participate}

This study was approved by the institutional review board of Kurashiki Central Hospital (approval number 2364). All patients gave their informed consent to participate in this study.

\section{Consent for publication}

Consent for publication was not required because all patient data were anonymised.

\section{Competing interests}

Tadashi Ishida has received honoraria from Pfizer, Japan, Inc. The other authors have no conflicts of interest to declare.

\section{Publisher's Note}

Springer Nature remains neutral with regard to jurisdictional claims in published maps and institutional affiliations.

\section{Author details}

${ }^{1}$ Department of Respiratory Medicine, Ohara Healthcare Foundation, Kurashiki Central Hospital, Miwa 1-1-1, Kurashiki, Okayama 710-8602, Japan. ${ }^{2}$ Department of Respiratory Medicine, Saiseikai Fukuoka General Hospital, Tenjinn 1-3-46, Chuoku, Fukuoka, Fukuoka 810-0001, Japan. ${ }^{3}$ Department of Respiratory Medicine, National Hospital Organization Minami Kyoto Hospital, Nakaashihara 11, Joyo, Kyoto 610-0113, Japan.
Received: 12 June 2017 Accepted: 7 December 2017

Published online: 16 December 2017

\section{References}

1. Ishida T, Tachibana H, Ito A, Tanaka M, Tokioka F, Furuta K, et al. Clinical characteristics of severe community-acquired pneumonia among younger patients: an analysis of 18 years at a community hospital. J Infect Chemother. 2014;20:471-6.

2. Amemura-Maekawa J, Kura F, Helbig JH, Chang B, Kaneko A, Watanabe Y, et al. Characterization of Legionella pneumophila isolates from patients in Japan according to serogroups, monoclonal antibody subgroups and sequence types. J Med Microbiol. 2010;59:653-9.

3. Helbig JH, Bernander S, Castellani P, Etienne J, Gaia V, Lauwers S, et al. PanEuropean study on culture-proven legionnaires' disease: distribution of Legionella pneumophila serogroups and monoclonal subgroups. Eur J Clin Microbiol Infect Dis. 2002;21:710-6.

4. Chen CY, Chen KY, Hsueh PR, Yang PC. Severe community-acquired pneumonia due to Legionella pneumophila serogroup 6. J Formos Med Assoc. 2006;105:256-62.

5. Lück PC, Schneider T, Wagner J, Walther I, Reif U, Weber S, et al. Community-acquired legionnaires' disease caused by Legionella pneumophila serogroup 10 linked to the private home. J Med Microbiol. 2008;57:240-3

6. Furugen $M$, Koide $M$, Baba M, Sato $Y$, Teruya $H$, Naha $Y$, et al. Legionella pneumonia caused by Legionella pneumophila serogroup 2: second case report in Japan. J Infect Chemother. 2008:14:161-5.

7. Kawanami T, Yatera K, Fukuda K, Yamasaki K, Kunimoto M, Nagata S, et al. Diagnosis of fulminant pneumonia caused by Legionella pneumophila serogroup 8 with the sequence analysis of the $16 \mathrm{~S}$ rRNA gene. Tohoku J Exp Med. 2011;225:65-9.

8. Grottola A, Forghieri F, Meacci M, Fabio A, Pozzi L, Marcheqiano P, et al. Severe pneumonia caused by Legionella pneumophila serogroup 11, Italy. Emerg Infect Dis. 2012;18:1911-3.

9. Faris $\mathrm{B}$, Faris $\mathrm{C}$, Schousboe $\mathrm{M}$, Heath $\mathrm{CH}$. Legionellosis from Legionella pneumophila serogroup 13. Emerg Infect Dis. 2005;11:1405-9.

10. Ito A, Ishida $T$, Tachibana $H$, Ito $Y$, Takaiwa $T$, Fujii $H$, et al. A case of community-acquired pneumonia due to Legionella pneumophila serogroup 9 in which initial treatment with single-dose oral azithromycin appeared useful. Jpn J Infect Dis. 2017; https://doi.org/10. 7883/yoken.JJID.2016.548.

11. Fiumefreddo R, Zaborsky R, Haeuptle J, Christ-Crain M, Trampuz A, Steffen I, et al. Clinical predictors for Legionella in patients presenting with community-acquired pneumonia to the emergency department. BMC Pulm Med. 2009;9:4

12. Haubitz $S$, Hitz F, Graedel $L$, Batschwaroff $M$, Wiemken $T L$, Peyrani $P$, et al. Ruling out Legionella in community-acquired pneumonia. Am J Med. 2014; 127(1010):e11-9.

13. Mandell LA, Wunderink RG, Anzueto A, Bartlett JG, Campbell GD, Dean NC, et al. Infectious Diseases Society of America/American Thoracic Society consensus guidelines on the management of community-acquired pneumonia in adults. Clin Infect Dis. 2007:44(Suppl 2):S27-72

14. American Thoracic Society; Infectious Diseases Society of America. Guidelines for the management of adults with hospital-acquired, ventilatorassociated, and healthcare-associated pneumonia. Am J Respir Crit Care Med. 2005;171:388-416.

15. Fine MJ, Auble TE, Yealy DM, Hanusa BH, Weissfeld LA, Singer DE, et al. A prediction rule to identify low-risk patients with community-acquired pneumonia. N Engl J Med. 1997;336:243-50.

16. Lim WS, van der Eerden MM, Laing R, Boersma WG, Karalus N, Town Gl, et al. Defining community-acquired pneumonia severity on presentation to hospital: an international derivation and validation study. Thorax. 2003;58: 377-82.

17. Miyashita N, Matsushima T, Oka M, Japanese Respiratory Society. The JRS guidelines for the management of community-acquired pneumonia in adults: an update and new recommendations. Intern Med. 2006:45:419-28.

18. Cillóniz C, Ewig S, Polverino E, Marcos MA, Esquinas C, Gabarrús A, et al. Microbial aetiology of community-acquired pneumonia and its relation to severity. Thorax. 2011;66:340-6.

19. Doebbeling BN, Wenzel RP. The epidemiology of Legionella pneumophila infections. Semin Respir Infect. 1987;2:206-21. 
20. Hofflin JM, Potasman I, Baldwin JC, Oyer PE, Stinson EB, Remington JS Infectious complications in heart transplant recipients receiving cyclosporine and corticosteroids. Ann Intern Med. 1987;106:209-16.

21. Kugler JW, Armitage JO, Helms CM, Klassen LW, Goeken NE, Ahmann GB, et al. Nosocomial legionnaires' disease. Occurrence in recipients of bone marrow transplants. Am J Med. 1983;74:281-8.

22. Marston BJ, Lipman HB, Breiman RF. Surveillance for legionnaires' disease. Risk factors for morbidity and mortality. Arch Intern Med. 1994;154:2417-22.

23. Cunha BA, Burillo A, Bouza E. Legionnaires' disease. Lancet. 2016;387:376-85.

24. Miller AC. Early clinical differentiation between legionnaires' disease and other sporadic pneumonias. Ann Intern Med. 1979;90:526-8.

25. Sopena N, Sabrià-Leal M, Pedro-Botet ML, Padilla E, Dominquez J, Morera J, et al. Comparative study of the clinical presentation of Legionella pneumonia and other community-acquired pneumonias. Chest. 1998;113: 1195-200.

26. Yu H, Higa F, Hibiya K, Furugen M, Sato Y, Shinzato T, et al. Computed tomographic features of 23 sporadic cases with Legionella pneumophila pneumonia. Eur J Radiol. 2010;74:e73-8.

27. Sakai F, Tokuda H, Goto H, Tateda K, Johkoh T, Nakamura H, et al. Computed tomographic features of Legionella pneumophila pneumonia in 38 cases. J Comput Assist Tomogr. 2007;31:125-31.

\section{Submit your next manuscript to BioMed Central} and we will help you at every step:

- We accept pre-submission inquiries

- Our selector tool helps you to find the most relevant journal

- We provide round the clock customer support

- Convenient online submission

- Thorough peer review

- Inclusion in PubMed and all major indexing services

- Maximum visibility for your research

Submit your manuscript at www.biomedcentral.com/submit 